\title{
VERSO IL SUPERAMENTO DELLA TRADIZIONE. ROTTURA E CONTINUITÀ NELLA PROFESSIONE NOTARILE TRA ANTICO REGIME E PRIMO OTTOCENTO: IL CASO DI MILANO
}

\author{
TOWARDS THE OVERCOMING OF TRADITION. BREAKDOWN AND \\ CONTINUITY IN THE NOTARY PROFESSION BETWEEN THE ANCIENT \\ REGIME AND THE EARLY 19TH CENTURY: THE CASE OF MILAN
}

\author{
Stefania T. Salvi \\ Università degli Studi di Milano
}

\begin{abstract}
English: The essay intends to examine the characteristics of the Milanese notary profession in the delicate moment of transition from the ancient regime to the subsequent Napoleonic age, when the ideology of the Revolution, exported by the Napoleonic armies, involved, in the specific notary sector, the abandonment of the heterogeneous functions that had characterized the eighteenth-century notaries, in a continuous balance between 'private' and 'public', and the affirmation of a series of new, fundamental principles. With the "legal revolution" that Napoleon introduced in Italy in the field of professions, an unprecedented transformation was achieved, even in the notarial sector. The suppression of professional colleges and corporations put an end to the age-old monopoly of qualifications, now liberalized both in relation to private and public activities. Any distinction between noble and bourgeois professions was canceled with the formal birth of the liberal professions.

In particular, the case of Milan will be studied: Milan, after having experienced some important changes at the end of the ancien régime, such as the birth of the public archive (1775), the Joseph II's reform of the recruitment of notaries and the Regolamento generale per i notari della Lombardia austriaca (1794), lived, first as the capital of the Napoleonic Kingdom of Italy and then in the political reality of the Lombardo-Venetian Kingdom, an intense season of lively debates on the opportunity and methods of innovating a profession, for some time exercised in a protean manner by a class, anything but compact, in which very different operators were distinguished from each other in terms of culture and social origin.

The Regolamento sul Notariato of the Kingdom of Italy, published on 17 June 1806, took up the discipline of the Regolamento generale per i notari della Lombardia austriaca and differed in some points from the French notarial law. Still in force after the fall of Napoleon, the Regolamento sul Notariato of 1806 was also an alternative model to French law in the Kingdom of Lombardy-Venetia, where, for a certain period, there was discussion on whether to maintain or abolish the notary profession. The Austrian civil code, applied in the Lombardo-Venetian Kingdom, did not regulate the figure of the notary by law and the Austrian domination did not leave great autonomy to the notaries.
\end{abstract}

* Italian Review of Legal History, 7 (2021), n. 23, pagg. 737-762

* https://riviste.unimi.it/index.php/irlh/index

* ISSN 2464-8914 - DOI 10.54103/2464-8914/16908. Articolo pubblicato sotto Licenza CC-BY. 
However, without apparently suffering the frenetic political upheavals of the first half of the nineteenth century, the Lombard notaries proved to be an industrious category, capable of adapting to the new times, no longer held back in their social and professional rise by the constraints imposed by the ancient society regime.

Despite the numerous studies conducted, in recent and less recent times, on notaries in the modern age, the subject is far from exhausted and, thanks to the rich documentary heritage preserved in the archives, it is possible and desirable to expand research on the specific reality of the nineteenth-century Lombard notary, which, in a period so full of changes, was going through an important process of professionalization. This process, after the Restoration, was defined in a precise manner and notaries became fully qualified professionals capable of aspiring to the most important city offices.

Keywords: notary profession; ancient regime; 19th Century; Milan; Lombardy

Abstract Italiano: II contributo intende esaminare i caratteri del notariato milanese nel delicato momento di passaggio dall'antico regime alla successiva età napoleonica, quando l'ideologia della Rivoluzione, esportata dalle armate napoleoniche, comportò, nello specifico settore notarile, l'abbandono delle eterogenee funzioni che avevano caratterizzato i notai settecenteschi, in continuo bilanciamento tra 'privato' e 'pubblico', e l'affermarsi di una serie di nuovi, fondamentali principi. Si studierà, in particolare, il caso di Milano che, dopo aver conosciuto alcuni cambiamenti di rilievo sul finire dell'ancien régime, come la nascita dell'Archivio pubblico (1775), la riforma giuseppina del reclutamento dei notai e il Regolamento generale per i notari della Lombardia austriaca (1794), visse, prima da capitale del Regno d'Italia napoleonico e poi nella realtà politica del Regno Lombardo-Veneto, un'intensa stagione di vivaci dibattiti in merito all'opportunità e alle modalità di innovare una professione, da tempo esercitata in maniera proteiforme da un ceto, tutt'altro che compatto, in cui si distinguevano operatori molto diversi tra loro per cultura e provenienza sociale. Senza apparentemente soffrire i frenetici rivolgimenti politici della prima metà dell'Ottocento, i notai lombardi si dimostrarono una categoria operosa, capace di adattarsi ai tempi nuovi, non più frenati, nella loro ascesa sociale e professionale, dai vincoli imposti dalla società di antico regime.

Parole chiave: notariato; antico regime; XIX secolo; Milano; Lombardia

Sommario: 1 . Premessa - 2. II notariato alla fine dell'antico regime e le riforme asburgiche -3 . L'età napoleonica e la Restaurazione -4 . Conclusioni.

\section{Premessa}

Al centro delle pagine che seguono sarà l'obiettivo di esaminare i caratteri del notariato milanese nel delicato momento di passaggio dall'antico regime alla successiva età napoleonica, nel tentativo di individuare i principali mutamenti che investirono la fisionomia del notaio, trasformandola in maniera decisiva, senza tralasciare come tale trasformazione fosse iniziata, irreversibilmente, già durante la prima dominazione austriaca in Lombardia. 
Le riforme asburgiche attuate in questi territori sul finire del XVIII secolo, imponendo il requisito della laurea in legge, cominciarono a erodere progressivamente il monopolio esercitato dai Collegi sull'accesso alla professione notarile, dando un primo, fondamentale scossone al tradizionale sistema di cooptazione a favore del concetto di 'accesso per merito', che si stava affacciando, in quei decenni, alle menti e alle coscienze europee. Fino alla fine del Settecento è, inoltre, testimoniata una profonda dicotomia tra professione 'privata' e dimensione pubblica dei notai incardinati presso diverse magistrature, attive a Milano e nel contado, e presso vari organismi, come la Curia arcivescovile che cooptava i propri notai secondo regole non sempre rispondenti ai criteri del Collegio cittadino.

Su questo panorama si abbatterono le riforme francesi, che soppressero le istituzioni corporative e ridussero il numero dei notai sulle singole piazze, consolidando la posizione di coloro che avevano la possibilità di esercitare il notariato.

Dopo aver inquadrato l'organizzazione della professione nella tarda età moderna, ricordando quali furono i principali interventi austriaci in materia e la molteplicità di funzioni e attività, che contraddistinsero i notai milanesi, si cercherà di comprendere, attraverso l'imprescindibile ausilio delle fonti, se tali caratteri furono davvero definitivamente superati con i cambiamenti portati dal XIX secolo.

\section{II notariato alla fine dell'antico regime e le riforme asburgiche}

Tra gli aspetti di maggiore interesse si segnalano le funzioni proteiformi che lo sfaccettato ceto notarile milanese fu in grado di svolgere, nel corso dell'età moderna, avvicendandosi con perizia e abilità tra compiti privati e pubblici.

Complice il progressivo declino del prestigio e del potere ${ }^{1}$ della categoria che, nel passaggio dal medioevo all'età moderna, subì un deciso ridimensionamento del suo ruolo, ad un primo sguardo i notai di antico regime sembrano immersi soprattutto nell'attività di certificazione della volontà negoziale dei privati. Tuttavia, uno studio più attento delle fonti a disposizione rivela l'importanza della dimensione 'pubblica' del notariato di questo periodo.

Com'è noto, al periodo d'oro dell'età comunale, in cui i notai «assunsero e seppero conservare, attraverso le loro preziosissime competenze, un ruolo di preminenza sociale e di contiguità immediata con le élites dirigenti» ${ }^{2}$, seguirono i secoli dell'età moderna che, in questo settore, fu caratterizzata da una forte discontinu-

\footnotetext{
1 II notaio, chiamato dal Comune ad autenticare gli atti e a fornire quella credibilità che la nuova associazione non poteva ancora attribuire direttamente, rivesti, in età comunale, una condizione di particolare prestigio, così come, in certi contesti, ad esempio a Bologna, riuscì a raggiungere le leve del potere. Cfr. Costamagna, 1970; Costamagna, 1978, pp. 559-565. Ma si veda altresi Fasoli, 1968, pp. 25-39; Fasoli, 1977, pp. 121-142; Fissore, 1989, pp. 99-128; Fissore, 1999, pp. 47-56. Più di recente v. Grillo, 2017, pp. 99-114.

2 Tavilla, 2013, pp. 143-180, in particolare p. 143. Sul ruolo dei notai in età comunale basti qui citare Ascheri, 2004, pp. 113-125; di Renzo Villata, 2009, pp. 15-64, in particolare pp. 22 ss.
} 
ità rispetto al passato. A partire dal Cinquecento, venuta meno la veste politica di questi professionisti, vi fu una riconversione sempre più marcata all'esercizio della professione privata. $E$ tale elemento costituisce forse una delle ragioni che ha indotto gli storici, soprattutto in passato, a manifestare scarso interesse per $i$ notai di questo periodo. Se la documentazione notarile è stata oggetto di numerosi studi, la figura del notaio, il suo specifico profilo professionale e la rilevanza del ruolo che, ancora in piena età moderna, era chiamato a giocare nel funzionamento della macchina amministrativa hanno sino a poco tempo fa suscitato una limitata attenzione, benchè questi operatori del diritto rappresentino un'importante componente anche della società di antico regime.

L'immagine che emerge dalle fonti relative alla specifica realtà lombarda è, infatti, quella di una categoria professionale estremamente versatile, tutt'altro che appiattita in un unico sbocco professionale, bensì connotata da una spiccata duttilità. A uno studio più attento, i notai settecenteschi si rivelano dotati di una straordinaria polivalenza, che consenti loro di incunearsi in molteplici settori istituzionali, dalle magistrature maggiori e minori al ricco entourage della Curia arcivescovile e dei luoghi pii ${ }^{3}$.

Tale eterogeneità si riscontra anche nel luogo di esercizio dell'attività professionale: dagli studi cittadini - che il più delle volte erano la casa di abitazione del notaio medesimo o la dimora del cliente - alla realtà delle campagne, ove i notai si recavano frequentemente per rispondere alle esigenze di una clientela a sua volta molto variegata ${ }^{4}$. Così, sia nel contado che in città, la professione poteva essere declinata in diversi ambiti, testimoniati dalle carte d'archivio, dalle quali emerge una polimorfa realtà notarile che, pur nel suo carattere composito, trova un comun denominatore nella figura del notaio inteso come 'professionista della scrittura'.

Ma occorre innanzitutto chiarire come fosse organizzato il notariato in quest'epoca.

Nel corso dell'età moderna l'attività dei notai e la loro stessa immagine pubblica si erano frammentate in una molteplicità di ruoli differenti, tanto che si potrebbe parlare di tanti gruppi di notai che, pur presentando inevitabili punti di contatto, erano variamente inseriti nelle molteplici strutture e nei diversi organismi della società d'ancien régime ${ }^{5}$.

Nello specifico contesto milanese, il notariato si articolava in tre compagini pro-

${ }^{3}$ Salvi, 2012a, passim.

4 Sulla diversificazione dei luoghi di redazione della documentazione notarile in età medievale v. Cancian - Fissore, 1992, pp. 81-109.

5 Salvi, 2012a; Salvi, 2017, pp. 213-230, in particolare p. 230. Al di fuori del contesto specificamente milanese, sulla estrema varietà dei regimi che, attraverso i secoli, regolarono l'esercizio del notariato nei diversi ordinamenti giuridici del territorio italiano v. di Renzo Villata, 2009, pp. 15-64; di Renzo Villata, 2016, pp. 131-152. 
fessionali, ordinate gerarchicamente: i pronotai, o secondi notai ${ }^{6}$, che avevano conseguito la laudatio tantum, i notai ad omnia laudati, titolari della laudatio ad omnia, e i causidici - oggi diremmo i procuratori legali, i rappresentanti delle parti in giudizio - che ottennero riconoscimento ufficiale negli statuti del XVI secolo e ai quali, a partire dal 1571, fu riservata la carica di Abati del Collegio notarile cittadino ${ }^{7}$.

Per divenire secondi notai occorreva superare un esame non molto complesso, mentre per poter entrare nel Collegio come ad omnia laudati ed essere così abilitati a rogare autonomamente, la selezione, riservata ai candidati in possesso di precisi requisiti, era ben più rigorosa.

L'operato del pronotaio (tantum laudatus o laudatus pro secundo notario) era soggetto a varie limitazioni nell'esercizio della professione: i pronotai non rogavano l'atto, né lo stendevano materialmente, ma si limitavano ad intervenire nel processo di elaborazione dei documenti che, in base agli statuti, richiedessero la loro presenza per accrescerne la solennità (ad esempio nei testamenti). Sovente i pronotai si accontentavano di lavorare presso lo studio di un notaio ad omnia laudatus senza mai conseguire la laudatio ad omnia, oppure esercitavano in un ufficio. Talvolta, dopo un adeguato periodo di formazione presso il banco di un causidico, il giovane pronotaio raggiungeva le competenze necessarie per affrontare l'esame e conseguire la laudatio ad omnia e, successivamente, il grado di causidico.

Quella dei notai ad omnia laudati era una categoria superiore rispetto a quella dei semplici pronotai, non soltanto per le maggiori possibilità di esercizio della professione e quindi di guadagno, ma soprattutto da un punto di vista sociale. Ricordiamo, infatti, che il mondo delle professioni legali in antico regime era fortemente gerarchizzato: esisteva una vera e propria 'piramide' verticale, il cui vertice era rappresentato dai nobili giureconsulti ${ }^{8}$.

I giudici del Senato di Milano, la più alta magistratura lombarda d'ancien régime, erano scelti esclusivamente tra i membri del Collegio dei giureconsulti, i quali erano cooptati nella struttura corporativa soltanto a condizione di appartenere al patriziato. Situazione analoga si riscontrava in altri Stati europei, ove, al livello superiore, si collocavano i giuristi attivi nelle grandi magistrature, la co-

\footnotetext{
${ }^{6}$ Secondo la ricostruzione di Roberto Perelli Cippo, a partire dai primi decenni del Duecento si diffuse a Milano la prassi di far presenziare, alla stesura di alcuni tipi di atti, oltre al rogatario altri notai. Man mano che la professione notarile andò organizzandosi in forme più precise, questo compito fu riservato a una categoria inferiore di notai, non ancora abilitati a rogare, e così il termine pronotarius, prima usato genericamente, entrò nella terminologia ufficiale (Perelli Cippo, 1982, pp. 594-598).

7 Dal 1571 la carica di Abate del Collegio dei notai fu riservata in maniera esclusiva a coloro che avessero ottenuto il grado di causidici collegiati da almeno quattro anni (Liva, 1979, pp. 205 ss.).

${ }^{8}$ Ci si limita qui a citare Padoa Schioppa, 1980, pp. 155-166; Padoa Schioppa, 2003, pp. 301-312; Pagano, 2001, pp. 355-418.
} 
siddetta nobiltà di toga (noblesse de robe), che affiancava la nobiltà tradizionale. I giureconsulti esercitavano, per inveterata tradizione, prestigiose prerogative come la facoltà di conferire le lauree e la potestas facere notarios.

Vi erano poi i giuristi di livello sociale inferiore, gli avvocati, i giudici delle magistrature cittadine, i causidici, i notai e i sollecitatori, diverse tipologie di operatori che componevano le fila della «folta turba» di professionisti del diritto descritta da Pietro Verri nell'intento di tratteggiare l'ambiente della pratica legale della Milano settecentesca9 ${ }^{9}$.

I notai, per l'appunto, si collocavano nei ranghi inferiori di tale gerarchia socio-professionale, che si basava non solo e non tanto sulle funzioni del giurista, bensì sulla sua appartenenza o meno al patriziato ${ }^{10}$. II declino sociale che il notariato attraversò in età moderna non fu però definitivo: la categoria seppe, infatti, riprendersi egregiamente nell'Otto-Novecento e la sua attuale prosperità - in Italia, così come nel resto d'Europa - lo dimostra.

Sebbene esistessero vistose differenze nella sfaccettata categoria dei notai di età moderna, tutt'altro che compatta, non possiamo ignorare i punti di contatto, gli elementi che, al contrario, accomunano i notai di antico regime: in primo luogo l'iscrizione al Collegio notarile cittadino, espressione, forse più che di un'autentica professionalità, di un preciso status sociale.

Notai e causidici facevano parte del medesimo organismo collegiale in alcune città lombarde, come Milano, Pavia, Como, Lodi, Cremona e Casalmaggiore, ove esisteva un Collegio dei notai e dei causidici, mentre a Mantova, così come a Vigevano, il collegio notarile non comprendeva i causidici ${ }^{11}$. A Sondrio, viceversa, non esisteva alcun collegio notarile: in Valtellina gli aspiranti notai non venivano cooptati all'interno di una corporazione, bensì accedevano alla professione previo esame delle loro qualità personali e capacità professionali da parte dei consoli di giustizia del territorio ${ }^{12}$. Il Collegio milanese tendeva poi ad esercitare un controllo stringente anche su realtà periferiche dello Stato di Milano, come, ad esempio, la Valsassina, ove pure esisteva un collegio notarile locale ${ }^{13}$.

Nel corso dell'età moderna l'ammissione all'esercizio della professione nel Ducato fu esclusiva prerogativa del Collegio, secondo le modalità di accesso descritte negli Ordini del Senato di Milano sull'esercizio della professione di notaio e cau-

\footnotetext{
9 Pietro Verri, Orazione panegirica sulla giurisprudenza milanese, in F. Venturi (ed.), Cesare Beccaria. Dei delitti e delle pene, con una raccolta di lettere e documenti relativi alla nascita dell'opera e alla sua fortuna nell'Europa del Settecento, Torino, Einaudi, 1970, pp. 144-145.

10 Cfr. Zorzoli, 2001, pp. 449-475. Vedi altresì Zorzoli, 2014, pp. 291-306.

11 Sugli interventi teresiani relativi al Collegio dei notai di Mantova si veda la documentazione conservata in Archivio di Stato di Milano (d'ora in poi ASMi), Studi parte antica, cartella 168, in particolare l'editto, a firma del conte Carlo di Firmian, datato 10 marzo 1764.

12 Mangini, 2005, pp. 149-194.

13 Dattero, 1997, pp. 155-167.
} 
sidico del 22 agosto $1686^{14}$. I requisiti previsti erano un'età minima di venticinque anni, la cittadinanza milanese o del Ducato, un reddito annuo di almeno cento scudi e la cosiddetta nobiltà negativa, ossia il mancato esercizio di arte vile ${ }^{15}$. Sul versante delle conoscenze professionali richieste, le norme si limitavano a esigere il compimento di un periodo di apprendistato obbligatorio, della durata di cinque anni, presso lo studio di un notaio collegiato e il superamento di una prova, consistente nella redazione di un instrumentum, estratto a sorte tra una serie di quindici atti differenti ${ }^{16}$.

Nel secondo Settecento, tuttavia, il Collegio notarile, così come tutte le strutture corporative osteggiate dall'assolutismo illuminato degli Asburgo, attraversava una fase di decadenza che preludeva alla sua definitiva scomparsa: la sua capacità di controllare l'accesso alla professione mediante le strategie di cooptazione messe in atto dagli Abati cominciò ad entrare in crisi sotto la pressione del processo riformistico avviato da Vienna.

Con ordine del 3 dicembre 1771 si impose l'obbligo, per tutti coloro che volessero esercitare il notariato, di conseguire la licenza presso l'Università di Pavia e di frequentare un corso di Arte notarile presso le Scuole Palatine di Milano ${ }^{17}$. Peraltro, il candidato privo di tali requisiti, ma in possesso del beneficium dispensationis Senatus, sarebbe stato ugualmente ammesso tra i notai collegiati. Qualche anno più tardi, il dispaccio teresiano dell'11 dicembre 1775 innalzava a ottocento lire annue, che dovevano provenire esclusivamente da beni immobili intestati al candidato, la rendita necessaria per l'immatricolazione ${ }^{18}$.

Questi provvedimenti tendevano a ridurre il numero dei notai, pur senza costituire una normativa organica in materia, come sarà il Regolamento generale per $i$ notari della Lombardia austriaca del 1794, cui si accennerà brevemente.

Sempre negli anni Settanta si realizzava, nel campo notarile, un'altra importante riforma, in grado di razionalizzare la conservazione degli atti rogati dai notai

${ }_{14}$ Ordines pro Notariis et Collegio Causidicorum, MDCLXXXVI, Die xxii Augusti, in Ordines Excellentissimi Senatus Mediolani ab anno MCDXC usque ad annum MDCXXXIX collecti, et scholiis ornati ab olim J.C. Angelo Stephano Garoni ..., Mediolani, in Curia regia sumptibus Joseph Richini Malatestae regii ducalis typographi, 1743, pp. 427-429, riprodotto anche in Giovanni Battista Bianchini, Compendium ordinum, stilatuum, et aliarum scripturarum decorem, et splendorem Ven. Collegii DD. Causidicorum et Notariorum Mediolani ostendentium, Mediolani, ex typographia Federici Francisci Maiettae, 1701, pp. 71-73.

${ }_{15}$ Su tale concetto cfr. Pino, 1979, pp. 339-378, in particolare pp. 357-359.

16 Il Fondo Collegio dei notai e dei causidici dell'Archivio di Stato di Milano conserva, divisi per anno, i fascicoli formati in occasione dell'ammissione dei candidati tra le fila dei pronotai, dei notai ad omnia laudati e dei causidici fino al 1803.

17 Sulle Scuole Palatine Visconti, 1922, pp. 3-14; Visconti, 1927; Scazzoso, 1982, pp. 887895; Guderzo, 1982, pp. 845-861; Brambilla, 1987, pp. 345-446, specialmente pp. 359366, pp. 386-398, pp. 422-430.

18 ASMi, Dispacci reali, cartella 254, dispaccio dell'11 dicembre 1775. 
defunti attraverso l'istituzione del Pubblico Archivio teresiano (1775) ${ }^{19}$.

L'antico sistema, che affidava la custodia degli atti, rogati dai notai defunti, a parenti che esercitassero la medesima professione ed era estremamente vantaggioso per i «possessori» di imbreviature ${ }^{20}$, tramontava definitivamente, compresso dalla macchina statale austriaca che, anche in questo ambito, avocava a sé il controllo dei meccanismi di conservazione della documentazione, trasformandoli in maniera radicale.

Se prima le carte notarili, anche quelle stilate dai notai ormai deceduti, erano serbate dai professionisti ancora in vita, per i quali costituivano una notevole fonte di guadagno - il rilascio di copie comportava, infatti, un compenso di non trascurabile entità - con la riforma teresiana del 1775 un profondo rinnovamento investì il mondo del notariato lombardo. L'Archivio pubblico non si poneva soltanto come nuova sede di registrazione degli atti, bensì assumeva l'importante funzione di conservare tutti i documenti fino ad allora sparsi disordinatamente presso i privati. II nuovo sistema scardinava, pertanto, le tradizionali modalità di conservazione della documentazione, a scapito di antichi privilegi. Consapevole del fatto che la riforma avrebbe comportato, per i notai milanesi, un danno di non lieve entità, il Collegio notarile, nella persona degli Abati, a più riprese supplicò - invano - l'Imperatrice di tenere in maggiore considerazione i diritti dei «possessori di imbreviature ${ }^{21}$.

Il braccio di ferro tra le autorità viennesi e il Collegio, sempre più sottoposto ad un penetrante controllo di impronta pubblicistica, proseguì anche negli anni successivi. Tuttavia, soltanto con gli interventi giuseppini del 1786 il governo centrale lo destitui dal suo tradizionale ruolo di indirizzo e controllo delle immatricolazioni, in ultima analisi di regolamentazione dell'accesso alla professione.

Con editto del 16 giugno 1786 si stabiliva che la domanda di ammissione al Collegio de' Notari e Causidici di Milano non dovesse più essere presentata al Collegio medesimo, bensì al Supremo Tribunale di giustizia, che avrebbe poi incaricato il Regio Tribunale d'appello di esprimere un parere circa la richiesta del petente. L'esame e l'approvazione di nuovi membri erano così sottratti alla corporazione per essere attribuiti al potere statale. II nuovo sistema faceva cadere

19 Salvi, 2009-2010, pp. 41-64.

20 Secondo gli statuti del Collegio notarile di Milano, redatti nel 1396, le imbreviature dei notai defunti dovevano essere trasmesse agli eredi che esercitassero la professione notarile e, qualora il rogatario defunto non ne avesse, spettava al Collegio designare il notaio collegiato che risultasse più idoneo. Prima che cominciasse a farsi strada l'idea di istituire un archivio pubblico, si guardava agli atti notarili come a un bene personale del notaio che li aveva rogati, cosicchè il notaio rogante poteva venderli e farne copia e tali diritti si trasferivano ai suoi eredi, ai quali, di regola, erano trasmesse le imbreviature del notaio defunto. Cfr. Confalonieri, 1965, pp. 161-198, in particolare pp. 185-186.

${ }^{21}$ ASMi, Uffici e tribunali regi parte antica, cartella 261, supplica degli Abati del Collegio dei notai e dei causidici di Milano Emanuele Giuseppe Airoldi e Giambattista Giletti (1773). 
le discriminazioni di nascita, che escludevano, ad esempio, il notaio laureato, ma nato da padre dedito al commercio, dalla possibilità di accedere alle magistrature civiche e regie riservate ai giureconsulti, quindi ai patrizi. Inoltre, con l'abolizione di tutte le giurisdizioni speciali, le autorità del Collegio notarile di Milano furono private della loro secolare competenza a giudicare nelle cause tra notai e terzi ratione notariae ${ }^{22}$.

Prima ancora dell'arrivo delle armate francesi guidate da Napoleone, Giuseppe II era riuscito ad esautorare l'antica struttura, privandola di ogni potere decisionale e riducendola ad un mero organo consultivo ${ }^{23}$.

Con I'ascesa al trono del fratello Leopoldo II, gli Abati del Collegio inviarono al nuovo imperatore una "lista delle osservazioni», in cui lamentavano, sdegnati, la privazione della giurisdizione criminale ${ }^{24}$ : la morte del secondogenito di Maria Teresa, sopraggiunta due anni dopo, impediva alle commissioni, nominate dal sovrano per la giustizia civile e penale, di terminare i lavori.

II Regolamento generale per i notari della Lombardia austriaca, emanato dall'Imperatore Francesco II nel $1794^{25}$, faceva chiarezza sulle qualità richieste al candidato, senza più ammettere deroghe: il petente doveva essere nato da legittimo matrimonio o legittimato per susseguente matrimonio, doveva avere l'età minima di venticinque anni ed essere suddito - per origine o in seguito a una permanenza di almeno dieci anni - della provincia del Collegio notarile in cui intendeva iscriversi. Agli aspiranti notai si richiedeva, inoltre, il conseguimento della laurea o della licenza presso I'Università di Pavia, da integrare con un tirocinio pratico, di almeno tre anni per il laureato e di quattro per il licenziato, presso un notaio e causidico collegiato di Milano ${ }^{26}$. Il requisito era ricordato anche nell'opera di Vincenzo D’Adda, professore di Arte notarile e di Istituzioni civili presso le Scuole Palatine ${ }^{27}$. Già alla fine del Settecento, quindi prima della riforma degli studi e delle professioni di matrice napoleonica, molti notai milanesi erano lau-

22 Liva, 1979, pp. 239-240. Sulla riforma giuseppina del sistema giudiziario ci si limita qui a ricordare Cuccia, 1971; Petronio, 1972, pp. 375 ss.

${ }^{23}$ Cfr. Brambilla, 1996, pp. 393-433, in particolare pp. 402-405; Levati, 2000, pp. $120-$ 151, in particolare p. 125.

${ }^{24}$ ASMi, Studi parte antica, cartella 169, Lista delle osservazioni, 20 giugno 1790.

25 ASMi, Studi parte antica, cartella 166, Regolamento generale per i notari della Lombardia austriaca, 18 marzo 1794. Il testo del provvedimento, sottoscritto da Ferdinando Governatore della Lombardia austriaca, è consultabile anche in Gridario dal 1727 al 1794, Milano, G.R. Malatesta [1727-1794], vol. VIII.

${ }^{26}$ Regolamento generale per i notari della Lombardia austriaca, 18 marzo 1794, paragrafo IV.

27 Vincenzo D'Adda, Arte notarile in tre parti divisa, Milano, Presso Giuseppe Taglioretti al Cordusio, 1796, t. I, Regolamento generale per i notari della Lombardia austriaca, IIIIV. Sulla vita e I'opera del notaio e causidico milanese Vincenzo D'Adda cfr. Salvi, 2012b; Salvi, 2013b, p. 624. 
reati ${ }^{28}$, benchè il requisito della laurea sia stato introdotto ufficialmente soltanto con la riforma del $1913^{29}$. Nemmeno nella regolamentazione del 1794 mancava poi la previsione del non esercizio di "arte vile, o ignobile nel comune concetto» sia da parte del candidato che del padre e dell'avo, così come era richiamato il più recente requisito del reddito minimo di ottocento lire milanesi, introdotto da Maria Teresa nel 1775.

In presenza di tutti gli elementi previsti dalla normativa e del voto favorevole, espresso dagli Abati sulla domanda di ammissione, si procedeva all'esame del candidato, consistente in una prova scritta e in un colloquio orale ${ }^{30}$.

In antico regime, così come era stato nei secoli medievali, i notai, da sempre interpreti delle esigenze giuridiche del loro tempo, furono straordinari veicoli dell'integrazione sociale, interlocutori di diverse istituzioni e del potere politico, partecipi com'erano non solo della redazione di documenti dotati di pubblica fede, destinati a testimoniare efficacemente le articolate vicende patrimoniali e non del Ducato di Milano ${ }^{31}$, bensì pure della preliminare contrattazione tra le parti, di cui erano sovente arbitri accorti.

Immerso nella vita sociale e politica della comunità, il notaio, come il prete e il medico, era persona di indubbio prestigio all'interno della comunità e giocava un ruolo importante nel mediare i contrapposti interessi di soggetti in conflitto tra loro $^{32}$. I notai del contado, che spesso appartenevano a influenti famiglie del luogo, erano ai vertici della gerarchia sociale, ricoprendo incarichi di rilievo nell'ambito dell'amministrazione locale. La rispettabilità sociale di cui godevano, unita all'opportunità di accedere $a$ incarichi autorevoli, che procuravano un certo consolidamento patrimoniale, rendeva quindi allettante l'accesso al locale Collegio notarile. È indubbio, infatti, che la qualifica di notaio potesse consentire e facilitare l'assunzione di cariche di varia natura, come quella di sindaco e cancelliere, così come di amministratore e tesoriere di diversi luoghi pii.

Come si diceva in apertura, la pluralità di attribuzioni, talvolta assai distanti tra loro, riscontrabili nei percorsi professionali di non pochi notai, costituisce un aspetto tutt'altro che privo di interesse, sinora poco approfondito, del notariato di questo periodo, che consente di rivalutare il versante pubblico della professione nella tarda età moderna. Accanto all'ambito propriamente privato, in cui il notaio poneva le sue competenze al servizio del cliente in vista della stesura di

\footnotetext{
28 Salvi, 2012a, pp. 50-51.

${ }^{29}$ Santoro, 1998; Santoro, 2004, pp. 313-314.

${ }^{30}$ Regolamento generale per i notari della Lombardia austriaca, 18 marzo 1794, paragrafo XIV.

31 Basta una rapida scorsa agli inventari del Fondo Notarile conservato presso l'Archivio di Stato di Milano per rendersi conto dell'immensa mole di materiale documentario a disposizione degli studiosi per quanto concerne il XVIII secolo.

32 Cfr., per la realtà veneta, Faggion, 2014, pp. 291-304. Per la Lombardia mi sia consentito rinviare a Salvi, 2022, in corso di stampa.
} 
un documento che rispecchiasse il più possibile la volontà espressa dalle parti, vi era, infatti, il profilo per così dire pubblico, in cui il notaio svolgeva le sue mansioni di certificatore all'interno di un ufficio o al servizio di un'istituzione.

A questo proposito era possibile individuare, all'interno della categoria dei notai ad omnia laudati, delle vere e proprie élites che operavano per le più alte magistrature, come il Regio Ducal Magistrato Camerale, un tempo suddiviso in Magistrato delle rendite ordinarie e Magistrato delle rendite straordinarie, che si occupava dell'amministrazione finanziaria dello Stato.

I notai camerali - così si chiamavano i notai di questa magistratura, pochi di numero e in qualche modo privilegiati -, forti di questo incarico e del prestigio che ne derivava, spesso consolidavano l'attività professionale privata. Si trattava di notai regolarmente iscritti al Collegio notarile che, in qualità di funzionari della Regia Camera, stendevano atti e sentenze del Magistrato dei redditi, secondo soltanto al potente Senato di Miano ${ }^{33}$. Tale documentazione, che il notaio rogava con l'assistenza di un coadiutore, costituisce la preziosa testimonianza delle molteplici operazioni economiche realizzate dalla Magistratura Camerale, tra cui soprattutto vendite, livelli e appalti, relativi alle attività più disparate, dai lavori di riparazione delle mura cittadine alla costruzione di nuovi ponti, ad altre operazioni di manutenzione da compiersi nelle diverse città lombarde, ad azioni più particolari, come la somministrazione del pane ai detenuti nelle regie carceri di Milano. Tra i rogiti camerali del secondo Settecento si incontrano numerosi documenti aventi ad oggetto i palchi di famosi teatri, assai ambiti dalle élites lombarde. Il possesso di un palco nei più importanti teatri milanesi, come il Regio Ducal Teatro - poi Teatro alla Scala - e il Teatro in contrada Larga rappresentava un vero e proprio status symbol, fonte al contempo di piacevole svago e di crescita culturale. I palchi teatrali erano oggetto prevalentemente di acquisti, cessioni e transazioni. Era poi sempre la Regia Camera a concedere appalti per l'ampliamento e la ricostruzione di vecchi teatri, così come per la realizzazione di opere teatrali $i^{34}$. L'interesse nutrito dall'amministrazione austriaca verso gli spettacoli teatrali è cosa nota, soprattutto in età teresiana ${ }^{35}$ : i palchi teatrali riempiono, quindi, buona parte dei rogiti camerali della seconda metà del Settecento, così come, negli stessi anni, furono al centro di una serie di vertenze giudiziarie ${ }^{36}$.

Simili, per certi aspetti, ai notai camerali erano i notai arcivescovili, che operavano per la Curia ambrosiana e appartenevano a un collegio interno alla Curia stess $^{37}$. Anche in questo caso spesso si trattava di notai che, parallelamente,

${ }^{33}$ La copiosa documentazione rogata per il Magistrato Camerale di Milano è conservata nel Fondo Rogiti camerali dell'Archivio di Stato di Milano.

34 Salvi 2012a, pp. 219-273.

${ }^{35}$ Cfr. Faron, 2000, pp. 49-73. Sul periodo successivo si veda altresì Levati, 1997, pp. 216-219.

${ }^{36}$ di Renzo Villata, 2006, pp. 1-117.

${ }^{37}$ Cfr. Belloni e Lunari (ed.), 2004. 
coltivavano una fiorente professione privata in grado di attrarre, come principali clienti, chiese e luoghi pii, a testimonianza del fatto che il notaio, collaborando con l'Arcivescovo, entrava in contatto e stringeva una serie di relazioni con persone ed enti più o meno direttamente collegati all'ambiente religioso. La specifica pratica nella redazione degli atti relativi all'ingente patrimonio ecclesiastico e la funzione di tramite con la Curia diocesana fecero sì che i notai arcivescovili divenissero un punto di riferimento quasi obbligato per tutti gli enti ecclesiastici della diocesi. Nell'area milanese di fine ancien régime il notarius curiae era un professionista di regola abilitato all'esercizio dell'attività secondo i modi consueti, iscritto alla matricola cittadina, che, in un secondo tempo, veniva scelto dalla Curia, in base a criteri essenzialmente fiduciari, dopo aver superato l'esame di abilitazione dinanzi al Collegio dei notai della Curia arcivescovile, organismo, retto dal Vicario generale, autonomo e separato dal Collegio notarile cittadino. Benchè l'esercizio del notariato fosse stato più volte interdetto agli ecclesiastici, dall'analisi delle fonti emergono numerosi casi di preti-notai. Sul punto la dottrina di diritto comune giunse a conclusioni differenti, mentre la giurisprudenza del Senato di Milano era stata inflessibile nel privare dell'ufficio notarile quanti indossassero la veste talare e in tal senso si espresse anche Maria Teresa d'Austria che, in un dispaccio del 1774, ribadì il divieto, per i chierici, di esercitare attività temporali estranee o disdicevoli al loro carattere, tra cui anche l'avvocatura, la medicina e la chirurgia ${ }^{38}$. A un livello decisamente più basso troviamo gli attuari o notai di tribunale, che potevano essere notai ad omnia laudati o semplici pronotai, la cui presenza è sistematicamente attestata non soltanto nei tribunali, ove spesso svolgevano le mansioni del giudice ${ }^{39}$, bensì pure a capo delle squadre degli uomini armati di podestà e capitani di giustizia che pattugliavano il territorio. Un gruppo estremamente eterogeneo dai contorni non sempre definiti, che, tuttavia, svolse un ruolo fondamentale nel funzionamento delle magistrature lombarde.

II non facile inquadramento di questa «massa anonima e sterminata di oscuri minutanti di curia» - secondo la definizione di Mario Montorzi ${ }^{40}$ - deriva principalmente dall'assenza, quantomeno nello Stato di Milano, di una disciplina generale valida per tutti i notai $a d$ acta: la normativa di riferimento appare, in questo più che in altri settori, dispersiva, sovrabbondante e talvolta contraddittoria e la molteplicità di compiti cui erano preposti gli attuari variava in base alla magistratura in cui erano incardinati.

Alcuni dispacci teresiani si erano occupati della categoria, affrontando soprattutto la vexata quaestio della loro remunerazione ${ }^{41}$ : gli attuari lamentavano, in-

\footnotetext{
38 ASMi, Dispacci reali, cartella 251, dispaccio del 14 febbraio 1774.

39 Da ultimo Sinisi, 2020, pp. 221-247.

40 Cfr. Montorzi, 1983, pp. 1090-1128; Montorzi, 1997, pp. 132-141. Ma si vedano anche gli studi di Lorenzo Sinisi: Sinisi, 2006, pp. 215-240; Sinisi, 2008, pp. 1039-1056; Sinisi, 2014, pp. 102-115; Sinisi, 2020, pp. 221-247.

41 ASMi, Giustizia punitiva parte antica, cartella 5; ASMi, Dispacci reali, cartella 202;
} 
fatti, una perenne indigenza, intollerabile - a leggere le accorate suppliche di alcuni di essi ${ }^{42}$ - considerata la qualità e quantità delle mansioni cui erano preposti all'interno delle cancellerie dei tribunali, così come all'esterno, impegnati com'erano nel controllo dell'ordine pubblico sotto la supervisione del capitano di giustizia e del podestà. Ben lontani dal prestigio che, ancora nel XVIII secolo, ammantava i notai camerali e arcivescovili, gli attuari non erano per nulla stimati dalla popolazione locale, non di rado bersaglio della loro cattiva condotta: si contano, infatti, numerosi casi di corruzione degli attuari, spesso inclini alla violenza e ad abusare dei propri poteri ${ }^{43}$.

A prescindere dal giudice con il quale collaborassero, compito principale di questi notai restava la verbalizzazione e la conservazione della documentazione giudiziaria, secondo una prassi che si tramandava dall'età medievale.

Le funzioni degli attuari vennero meno soltanto all'inizio del XIX secolo, quando la redazione della documentazione processuale fu definita dalla legge del tutto incompatibile con l'esercizio delle funzioni notarili.

\section{L'età napoleonica e la Restaurazione}

All'inizio dell'Ottocento la commistione di funzioni, che aveva a lungo caratterizzato il notaio di antico regime, aveva ormai i giorni contati.

Nel triennio giacobino e nei primi anni del XIX secolo il Collegio notarile continuò a funzionare con le ridotte prerogative che la riforma giuseppina non aveva intaccato, limitandosi ad approvare quanti erano già stati selezionati dal Tribunale d'Appello di Milano ${ }^{44}$. Pare, tuttavia, significativo riscontrare, nei processetti informativi redatti in occasione della presentazione al Collegio in questi anni, $\mathrm{i}$ certificati che attestano i «buoni costumi», la buona moralità dei candidati, a testimoniare la persistenza di alcuni retaggi del passato, nonostante il mondo stesse cambiando e le carte riportassero le rivoluzionarie parole «libertà» e «eguaglianza» ${ }^{45}$. Nel complesso, la documentazione, conservata dalle autorità collegiali, non risulta molto diversa da quella settecentesca: si ritrovano, infatti, fedi di battesimo degli aspiranti notai e fedi di matrimonio dei loro genitori, certificati di buona moralità a firma del curato del luogo di residenza, l'indicazione dei quesiti su cui si era svolto l'esame e l'attestato della pratica compiuta presso un notaio collegiato oltre, naturalmente, al verbale della "Commissione civile

\footnotetext{
ASMi, Dispacci reali, cartella 203, cartella 205 e cartella 209.

${ }^{42}$ ASMi, Giustizia punitiva parte antica, cartella 5, ricorso, datato 1769, dei notai Giuseppe Pintori e Antonio Morone; Ivi, risposta del capitano di giustizia Carlo Francesco Parravicini datata 6 maggio 1769.

43 Salvi, 2012a, pp. 458-500.

${ }^{44}$ Sulla nascita del Tribunale d'appello di Milano, presieduto dall'ex senatore Carlo Biondi, v. Cuccia, 1971, pp. 115 ss.; Capra, 1984, pp. 533-535.

${ }^{45}$ ASMi, Collegio dei notai e dei causidici, cartella 236, anno 1802.Pronotai, fascicolo di Paolo Antonio Bianchi.
} 
presso il Tribunale d'appello» che aveva esaminato il giovane.

La grande novità consisteva nel fatto che non fosse più il Collegio a selezionare i futuri rogatari che, una volta superato l'esame presso il Tribunale d'appello, si presentavano ai «Delegati al notariato in Milano» - non si parla nemmeno più di Abati - soltanto per prestare il consueto giuramento ${ }^{46}$.

Con la «rivoluzione legale ${ }^{47}$ che il dominio napoleonico introdusse al di qua delle Alpi nell'ambito delle professioni si preparava, anche nel settore notarile, una trasformazione senza precedenti. La soppressione dei collegi professionali e delle corporazioni metteva fine al secolare monopolio delle abilitazioni, ora liberalizzate sia relativamente all'attività privata che a quella pubblica. Cadeva, quindi, per usare le parole di Elena Brambilla, «ogni distinzione tra professioni nobili e borghesi» con la nascita formale della borghesia delle professioni liberali ${ }^{48}$. La riforma teresiana degli studi superiori, che per i notai aveva comportato l'istituzione di una cattedra di Arte notarile nelle Scuole Palatine, non aveva infatti pregiudicato il potere del Collegio milanese: indisturbata, la corporazione aveva così potuto continuare a escludere dall'esercizio del notariato sulla piazza di Milano i notai originari di altre città dello Stato - Pavia, Cremona, Como, Mantova con i rispettivi contadi - che, a loro volta, applicavano lo stesso principio e a distinguere tra notai e causidici collegiati e quelli soltanto approvati. La prima, significativa incrinatura si ebbe, come si è visto, soltanto con gli interventi giuseppini.

Nella Francia del Consolato furono introdotte una serie di discipline settoriali che limitavano il numero e l'ambito territoriale di diverse attività, tra le quali il notariato, disciplinato in modo più razionale e moderno con la legge del 25 Ventoso anno XI (16 marzo 1803) ${ }^{49}$. Tale provvedimento, dapprima direttamente operativo nei territori della Repubblica italiana di Napoleone Bonaparte e Francesco Melzi d'Eril, poi Regno d'Italia ${ }^{50}$, e nei territori annessi all'Impero francese, era destinato a sopravvivere ben oltre gli anni napoleonici, ispirando la regolamentazione della professione notarile, ormai definitivamente separata da quella forense, negli Stati italiani preunitari. Benché strutturata in modo più organico e ordinato, la legge del Ventoso seguiva gli orientamenti espressi nel decreto del 29 settembre- 6 ottobre $1791^{51}$, teso a superare una serie di problematiche e di abusi radicati nel tempo aspramente denunciati nei cahiers de doléances ${ }^{52}$.

A partire dal 1805, nel Regno d'Italia cominciavano i lavori di elaborazione di un

\footnotetext{
46 ASMi, Collegio dei notai e dei causidici, cartella 236, anno 1802.Notai e anno 1803. Notai.

47 Brambilla, 1997, pp. 153-208.

48 Brambilla, 1997, p. 203.

49 Sull'influenza che tale legge ebbe nel contesto italiano v. Sinisi, 2012, pp. 41-108, in particolare pp. 45-49.

50 Cfr. Zaghi, 1989.

51 Cfr. Mazzanti Pepe - Ancarani, 1983, pp. 73-92.

52 Mazzanti Pepe - Ancarani, 1983, pp. 38-53.
} 
regolamento sul notariato, sfociati nella pubblicazione della legge del 17 giugno $1806^{53}$, la cui disciplina, che in alcuni punti si discostava dalla legge del Ventoso, veniva armonizzata con il testo del Code Napoléon nell'opera del notaio e avvocato Francesco Maria Carcano ${ }^{54}$, già membro della commissione incaricata di elaborare lo stesso regolamento ${ }^{55}$.

Per certi aspetti, la disciplina napoleonica non faceva che riprendere il Regolamento austriaco del 1794: nelle disposizioni relative all'ammissione al notariato era, infatti, prevista la necessità della laurea, così come l'obbligo, per il candidato, di superare un esame scritto e orale, di cui doveva essere redatto un verbale ${ }^{56}$. $E$ proprio tale "efficace contaminazione fra alcuni elementi di matrice francese ed altri invece antichi di chiara derivazione italiana ${ }^{57}$ spiega il successo di questa normativa che, ancora vigente dopo la caduta di Napoleone, riuscì a porsi come modello alternativo alla stessa legge del Ventoso, con la conseguente apparizione di utili manuali teorico-pratici ad uso della categoria.

L'azione dello Stato investiva così il mondo delle professioni intellettuali, riformando gli studi superiori con l'intento di dare uniformità nazionale, sotto il controllo statale, alle carriere professionali, rinnovando percorsi formativi e sbocchi professionali.

Nell'Italia napoleonica - è noto - si sono poste le basi dell'Italia contemporanea, con la creazione di istituzioni prima sconosciute e con il delinearsi di nuove figure cetuali, come la burocrazia professionale, nel cui solco si sarebbero inseriti anche i notai.

Anche per l'Ottocento è possibile ricostruire le vicende di alcuni notai che riuscirono a coronare una felice parabola verso i vertici della società lombarda: si pensi, ad esempio, a Ignazio Baroggi, esponente di una famiglia di tradizione mercantile, che attraverso un'abile rete di relazioni parentali e matrimoniali divenne consuocero di Alessandro Manzoni, che era suo cliente - $\mathrm{fu}$ in grado di inserirsi nell'élite cittadina ambrosiana, come le ricerche di Stefano Levati hanno messo in luce ${ }^{58}$. Nell'ascesa di alcuni notai ottocenteschi non è difficile scorgere il ruolo decisivo giocato dallo Stato napoleonico e dalla mutata mentalità che, a differenza della rigida visione di antico regime, consentiva ora anche a chi ap-

53 Regolamento sul Notariato, 17 giugno 1806, Milano, dalla Stamperia Reale, 1806, altresì in Bollettino delle leggi del Regno d'Italia, Milano, 1805-1814, 1806, parte II, pp. 664-717. Cfr. Roberti, 1947, pp. 119-122; Mazzanti Pepe-Ancarani, 1983, pp. 175-203.

54 Francesco Maria Carcano, II Notajo istruito colli schiarimenti del regolamento sul notariato e delle leggi analoghe e colle module degl'istromenti ..., Milano, dalla Società Tipografica de' Classici Italiani, s.d. [1810]. Su Francesco Maria Carcano v. Salvi, 2013a, p. 444.

55 di Renzo Villata, 2016, pp. 131-152, in particolare p. 143.

56 Regolamento sul Notariato, 17 giugno 1806, Milano, dalla Stamperia Reale, 1806, p. 2, art. 14 c. 4 , p. 3, art. 18.

57 Sinisi, 2012, p. 55.

58 Levati, 2017, pp. 231-244. 
prodasse al notariato da realtà familiari meno prestigiose concrete possibilità di ascesa sociale e professionale.

Nonostante la volontà di addivenire ad un diverso regime $\mathrm{e}^{59}$, il Regolamento italico rimase operativo anche nei decenni successivi, fino all'entrata in vigore della prima legge notarile dello Stato unitario.

Con l'istituzione, nel 1815, del Regno Lombardo-Veneto ${ }^{60}$, si manteneva vigente la disciplina introdotta durante il regime napoleonico, benché il panorama giuridico coevo fosse ben lungi dal favorire l'affermazione e lo sviluppo della professione. La conservazione del Regolamento sul Notariato del 1806 appare, infatti, un'eccezione nel panorama legislativo del nuovo Stato che, in tutto dipendente da Vienna in quanto retto da un vicerè scelto tra i membri della famiglia imperiale, abolì immediatamente la codificazione napoleonica sostituendola con quella austriaca tradotta in italiano.

Negli anni della Restaurazione il dibattito sulla professione notarile fu intenso e nel $1817 \mathrm{ci}$ si domandava addirittura se mantenere o abolire il notariato ${ }^{61}$. ॥ codice civile austriaco, applicato in questi territori ${ }^{62}$, non disciplinava normativamente la figura del notaio, tanto che Daniele Lissoni, notaio in Milano, considerava che

Siccome in questo Codice non si fa menzione dei Notai, pareva che l'istituzione notarile dovesse cadere, e che i cittadini ai quali in ogni genere di transazioni civili era accordata la facoltà di valersi di scritture private, che avevano lo stesso vigore degli atti pubblici, avessero ad usare di tale libertà a risparmio, se non altro, di maggiori spese che l'ufficio del Notaio importava di conseguenza ${ }^{63}$.

Ma ciò naturalmente non avvenne: dei servigi dei notai si continuò a fare richiesta, come lo stesso Lissoni, tra gli altri, testimonia ${ }^{64}$.

Nel 1835 lo stesso Imperatore d'Austria proclamava «lo voglio che nel mio Regno Lombardo-Veneto debba sussistere ulteriormente il Notariato, con quegli attributi che corrispondono ai bisogni degli abitanti» ${ }^{65}$. Tuttavia, durante la seconda dominazione austriaca in Lombardia, una legge notarile, sostitutiva di quella d'ispirazione francese, non fu mai promulgata, nonostante i tentativi, compiuti negli

59 di Renzo Villata, 2009, pp. 15-64, in particolare pp. 55-57.

60 Meriggi, 1981a, pp. 207-245; Meriggi, 1981b, pp. 315-343; Meriggi, 1983; Meriggi, 1987; Meriggi, 2002, p. 125; Pontiggia e Rumi (ed.), 1988.

${ }^{61}$ Alcune notizie sul dibattito circa l'opportunità di conservare o abolire il notariato si ricavano dall'eterogenea documentazione conservata in ASMi, Studi parte moderna, cartella 319. In storiografia cfr. Mazzanti Pepe - Ancarani, 1983, pp. 270 ss.

62 Di Simone, 1994, pp. 1015-1038; Di Simone, 2010, pp. 291-317; di Renzo Villata, 2015, pp. 133-189; di Renzo Villata, in corso di stampa.

63 Daniele Lissoni, Progetto di legge per l'esercizio del Notariato con annotazioni, cenni storici e raffronti, Milano, Tip. Boniardi-Pogliani di Ermen. Besozzi, 1868, p. 9.

${ }^{64}$ Ibidem, p. 9. Cfr. di Renzo Villata, 2009, pp. 15-64, in particolare p. 56.

65 ASMi, Studi parte moderna, cartella 319, Risoluzione imperiale, 4 luglio 1835. 
anni Cinquanta, di estendervi i regolamenti vigenti per le antiche province della monarchia austriaca ${ }^{66}$.

Nel 1844 presso il governo lombardo-veneto fu sollevato il problema della compatibilità tra l'esercizio del notariato e l'impiego presso pubblici uffici («in un ufficio pubblico o comunale o presso di uno stabilimento di beneficenza»): dopo un ampio dibattito, la questione era risolta in senso affermativo, purchè tali funzioni non portassero il notaio ad abbandonare la propria residenza ${ }^{67}$. Pare significativo che, anche nella discussione di questa problematica, fosse richiamato il Regolamento del 1806 e specificamente il suo articolo 8, ove era chiaramente precisato che soltanto gli uffici di giudice di pace, di giudice, di cancelliere e sotto-cancelliere di un tribunale o di una corte, così come di regio procuratore, di ricevitore delle imposte, di commissario di polizia, di avvocato, di patrocinatore e di usciere fossero incompatibili con l'esercizio del notariato ${ }^{68}$. Allo stesso anno risale il Prospetto de' diversi Notaj che in Lombardia esercitano altre funzioni, dal quale si apprende che non pochi notai rivestivano contemporaneamente la carica di segretario o cancelliere di un luogo pio cittadino, così come di agente comunale e di agrimensore $^{69}$.

Benchè limitati nelle loro attribuzioni, non più estese come durante l'antico regime, i notai milanesi di questo periodo rogavano moltissimo: I'ingente mole di documenti conservati, per gli anni della Restaurazione, nel Fondo Notarile dell'Archivio di Stato di Milano testimonia, ancora una volta, l'operosità di una categoria che continuava a esercitare la professione senza apparentemente soffrire il frenetico susseguirsi dei cruciali avvenimenti politici sette-ottocenteschi.

Nel 1816 veniva steso un atto di costituzione di dote che, se non fosse per la data riportata dal rogatario e per il richiamo all'imperatore Francesco I, potrebbe essere stato redatto alla fine del Settecento. Si presentavano al cospetto del notaio Ambrogio Mangiagalli i coniugi Pasquale Colombo e Rosa Maria Pavesi insieme allo zio del defunto primo marito di lei, il quale confessava di aver costituito la dote della sposa, consistente in una determinata somma di denaro e nel corredo, per un totale di 2057 lire milanesi. Lo sposo, a sua volta, confessava di aver ricevuto, a nome della sposa "presente e accettante», i denari e gli oggetti elencati nella nota allegata ${ }^{70}$ e di avere assegnato alla consorte una controdote, composta

66 Mazzanti Pepe - Ancarani, 1983, pp. 275-277; Sinisi, 2012, pp. 79-82.

${ }^{67}$ ASMi, Studi parte moderna, cartella 320, decreto del 13 settembre 1844 e documentazione allegata.

${ }^{68}$ Regolamento sul Notariato, 17 giugno 1806, Milano, dalla Stamperia Reale, 1806, p. 2, art. 8.

${ }^{69}$ ASMi, Studi parte moderna, cartella 320, Prospetto de' diversi Notaj che in Lombardia esercitano altre funzioni.

${ }^{70}$ Come per il Settecento, anche nell'Ottocento gli atti dotali recano la «Nota della Scherpa». 
da 450 lire e da "gioie, vestiti e guanti di seta», per un totale di 650 lire milanesi ${ }^{71}$. Se in antico regime le doti occupavano un posto di assoluto rilievo nell'ambito della prassi notarile milanese, che registra un altissimo numero di «confessi» di doti matrimoniali, confermando l'importanza che, nei secoli, l'istituto dotale aveva sempre rivestito ${ }^{72}$, anche gli atti notarili della prima metà del XIX secolo testimoniano che la dote continuava ad essere profondamente radicata nella vita familiare ${ }^{73}$.

Negli anni precedenti e immediatamente successivi capita di imbattersi in simili convenzioni matrimoniali ${ }^{74}$ che, anche nell'aspetto esteriore e formale del documento, non paiono molto distanti dai rogiti del tardo antico regime ${ }^{75}$.

Anche per quanto concerne i testamenti, che non soltanto rappresentavano il principale strumento di trasmissione mortis causa della proprietà, ma servivano altresì a regolare i rapporti familiari, risolvendo questioni pregresse e prevedendo soluzioni future - come, ad esempio, la scelta, da parte del padre, del tutore e del curatore dei figli minori -, non si notano cambiamenti significativi né nelle clausole formali, né nel contenuto di questi atti ${ }^{76}$, che si confermano un punto d'osservazione privilegiato della società coeva ${ }^{77}$.

\section{Conclusioni}

Le fonti analizzate dimostrano che non vi era, nel Settecento, un tipo univoco di notaio, bensì una serie di figure differenti, che costellavano, ciascuna con le proprie specifiche competenze, il panorama di una professione in continuo divenire, che, ciclicamente, ha presentato momenti di ascesa e declino. II ceto notarile, sul finire dell'età moderna, presentava dunque molte sfaccettature, dimostrandosi in grado di svolgere compiti anche molto diversi tra loro.

\footnotetext{
71 ASMi, Notarile, cartella 50558, notaio Mangiagalli Ambrogio quondam Luigi, 15 ottobre 1816, confesso di dote.

72 Cfr. Salvi, 2012a, pp. 147-166.

73 Per un quadro d'insieme ci si limita a rinviare a Lombardi, 2008.

74 Per una breve ricognizione nella prassi notarile lombarda dei primi anni della Restaurazione cfr. di Renzo Villata, 2015, pp. 174-181.

75 Permane, anche nell'Ottocento, la dicitura «confesso di dote», tipica degli atti di costituzione di dote della seconda metà del Settecento, quando progressivamente scompariva la lingua latina e gli atti notarili erano ormai quasi sempre stesi in lingua italiana. Si pensi poi alla formula di chiusura «Fatto, letto e celebrato nella casa di abitazione di me notaro infrascritto", con cui il notaio attestava la stesura dell'atto alla presenza di due testimoni «idonei, noti e conoscenti delle parti contraenti».

${ }^{76}$ In merito a tipologie, struttura e contenuti del testamento settecentesco mi permetto di richiamare ancora Salvi, 2012a, pp. 182-216.

77 Cfr., a mero titolo esemplificativo, ASMi, Notarile, cartella 46551, notaio Maggi Gaetano quondam Benedetto, 16 aprile 1817, testamento di Bartolomeo Ceroni; Ivi, 30 maggio 1817, testamento di Teresa Stroppa vedova Croce.
} 
Non soltanto, dunque, il notaio tradizionalmente impegnato nella più nota libera professione, legato esclusivamente alla scrittura di contratti e testamenti, bensi tanti professionisti che, pur accomunati da inevitabili punti di contatto, erano variamente inseriti nelle molteplici istituzioni della realtà cittadina ed extraurbana.

Per un definitivo cambiamento nel modo di percepire i mestieri, e tra questi il notariato, occorreva attendere la temperie napoleonica, che, con la legge del Ventoso e il Regolamento del 1806, cancellava, almeno in parte, la versatilità dei notai di antico regime e la loro straordinaria capacità di adattarsi alle esigenze della prassi e del pluralismo istituzionale, ancora nettamente avvertibile alla fine del Settecento ${ }^{78}$.

Tuttavia, nonostante la soppressione del Collegio e i mutamenti politici che investirono la Lombardia nei primi decenni del XIX secolo, i notai non sembrano, almeno in apparenza, soffrire particolarmente il cambiamento e alcuni caratteri della prassi notarile di antico regime perdurano pressochè inalterati: mi riferisco, in particolare, alla struttura e ai contenuti di alcuni atti notarili che, ad una prima analisi, condotta necessariamente a campione, appaiono mutare soltanto negli aspetti più formali.

Ulteriori ricerche andrebbero svolte sulla specifica realtà del notariato lombardo ottocentesco, che, in un periodo così denso di rivolgimenti, si stava adattando ai tempi nuovi, non più frenato, nella sua ascesa, dai vincoli imposti dal patriziato in età moderna, bensì pronto a entrare a pieno titolo nell'agone delle cariche cittadine sulla base di un processo di professionalizzazione che, dopo la Restaurazione, fu definito con rigore ${ }^{79}$.

\section{Bibliografia}

Ascheri M., 2004: I problemi del successo: i notai nei comuni tardo-medievali italiani, in Aragón en la Edad Media. Perspectivas actuales sobre las fuentes notariales de la Edad Media, Zaragoza, Universidad de Zaragoza, pp. 113-125

Belloni C., Lunari M. (eds.), 2004: I notai della Curia arcivescovile di Milano (secoli XIV-XV), Roma, Ministero per i beni e le attività culturali

Brambilla E., 1987: Le professioni scientifico-tecniche a Milano e la riforma dei collegi privilegiati (sec. XVII-1770), in G. Barbarisi (ed.), Ideologia e scienza nell'opera di Paolo Frisi (1728-1784), Atti del Convegno internazionale di studi (Politecnico di Milano, 3-4 giugno 1985), vol. I, Milano, FrancoAngeli, pp. 345446

78 Sulle leggi ottocentesche relative all'ordinamento del notariato cfr. Sinisi (ed.), 2011.

79 Tra gli studi recenti relativi ad altre realtà territoriali dell'Italia del primo Ottocento v. De Renzi, 2015. 
Brambilla E., 1996: Libertà filosofica e giuseppinismo. Il tramonto delle corporazioni e l'ascesa degli studi scientifici in Lombardia, 1780-1796, in G. Barsanti, V. Becagli, R. Pasta (ed.), La politica della scienza. Toscana e Stati italiani nel tardo Settecento, Atti del Convegno di Firenze 27-29 gennaio 1994, Firenze, Olschki, pp. 393-433

Brambilla E., 1997: Università, scuole e professioni in Italia dal primo Settecento alla Restaurazione. Dalla "costituzione per ordini» alle borghesie ottocentesche, in "Annali dell'Istituto storico italo-germanico in Trento", 22, pp. 153-208

Cancian P., Fissore G.G., 1992: Mobilità e spazio nell'esercizio della professione notarile: l'esempio dei notai torinesi (secc. XII-XIII), in "Bollettino storicobibliografico subalpino", 90, pp. 81-109

Capra C., 1984: /l Settecento, in D. Sella, C. Capra, // Ducato di Milano dal 1535 al 1796, Storia d'Italia diretta da G. Galasso, vol. XI, Torino, Utet, pp. 153-617

Confalonieri P., 1965: I/ Collegio dei notai milanesi nel periodo visconteo-sforzesco, in "Acme", 18, pp. 161-198

Costamagna G., 1970: Il notaio a Genova tra prestigio e potere, Roma, Consiglio Nazionale del Notariato

Costamagna G., 1978: Notaio (diritto intermedio), in Enciclopedia del diritto, vol. XXVIII, Milano, Giuffrè, pp. 559-565

Cuccia S., 1971: La Lombardia alla fine dell'Ancien Régime. Ricerche sulla situazione amministrativa e giudiziaria, Firenze, La Nuova Italia

Dattero A., 1997: Il notariato di una comunità di valle dello Stato di Milano durante l'età moderna: aspetti istituzionali e sociali, in A. Pastore, M.L. Betri (ed.), Avvocati medici ingegneri. Alle origini delle professioni moderne, Bologna, Clueb, pp. 155-167

De Renzi I., 2015: L'élite sovversiva. I notai nello Stato pontificio dall'età giacobina all'Unità, Roma, Gangemi Editore

di Renzo Villata M.G., 2006: L'arte del difendere e l'allegare tra ancien régime ed età dei codici, in M.G. di Renzo Villata (ed.), L'arte del difendere. Allegazioni avvocati e storie di vita a Milano tra Sette e Ottocento, Milano, Giuffrè, pp. 1-117

di Renzo Villata M.G., 2009: Per una storia del notariato nell'Italia centrosettentrionale, in M. Schmoeckel e W. Schubert (ed.), Handbuch zur Geschichte des Notariats der europäischen Traditionen, Baden-Baden, Nomos, pp. 15-64

di Renzo Villata M.G., 2015: Tra bravi zelanti 'artigiani del diritto' al lavoro. L'introduzione dell'ABGB nel Lombardo-Veneto, con particolare riguardo alla Lombardia, in P. Caroni e R. Ferrante (ed.), La codificazione del diritto fra il Danubio e l'Adriatico. Per i duecento anni dall'entrata in vigore dell'ABGB (18122012), Atti del convegno internazionale (Trieste, 25-27 ottobre 2012), Torino, Giappichelli, pp. 133-189 
di Renzo Villata M.G., 2016: II notariato nell'Italia del Sette-Ottocento tra cultura giuridica e pratica, in M.T. Guerrini, R. Lupi, M. Malatesta (eds.), Un monopolio imperfetto. Titoli di studio, professioni, università (secc. XIV-XXI), Bologna, Clueb, pp. 131-152

di Renzo Villata M.G., L'application de l'ABGB dans le Royaume Lombard-Vénitien: premières issues d'une recherche, in Journées de la Société Internationale d'Histoire de Droit, Salzburg, in corso di stampa

Di Simone M.R., 1994: L'introduzione del codice civile austriaco in Italia. Aspettie momenti, in Scintillae luris. Studi n memoria di Gino Gorla, II, Milano, Giuffrè, pp. 1015-1038, ora in Ead., Percorsi del diritto tra Austria e Italia (secoli XVII$X X)$, Milano, Giuffrè, 2006, pp. 159-183

Di Simone M.R., 2010: Das ABGB in Italien, in Berger E. (ed.), Österreichisches Allgemeines Bürgerliches Gesetzbuch (ABGB). Eine europäische Privatrechtskodification, III, pp. 291-317

Faggion L., 2014: Social mediation in the Venetian regional state. The notary, the uncle, the priest (C. 1560-1590), in "Acta Histriae", 22, pp. 291-304

Faron O., 2000: Marché de prestige ou marché impossible? La distribution des loges de la Scala de Milan (1778-1930), in "Popolazione e Storia", I, nn. 1-2, pp. 49-73

Fasoli G., 1968: Giuristi, giudici e notai nell'ordinamento comunale e nella vita cittadina, in G. Rossi (ed.), Atti del Convegno internazionale di Studi Accursiani (Bologna, 21-26 ottobre 1963), vol. I, Milano, Giuffrè, pp. 25-39

Fasoli G., 1977: Il notaio nella vita cittadina bolognese (secc. XII-XV), in Notariato medievale bolognese, t. II, Roma, Consiglio Nazionale del notariato, pp. 121142

Fissore G.G., 1989: Alle origini del documento comunale. I rapporti fra i notai e I'istituzione, in Atti della Società Ligure di Storia Patria, 103, pp. 99-128

Fissore G.G., 1999: Il notaio ufficiale pubblico dei Comuni italiani, in P. Racine (ed.), Il notariato italiano del periodo comunale, Piacenza, Fondazione di Piacenza e Vigevano, pp. 47-56

Grillo P., 2017: Repubbliche di notai? II ruolo politico del notariato nelle città italiane del secondo duecento, in P. Grillo e S. Levati (ed.), Legittimazione e credito tra medioevo e ottocento. Notai e ceto notarile tra ruoli pubblici e vita privata, Milano, FrancoAngeli, pp. 99-114

Guderzo G., 1982: La riforma dell'Università di Pavia, in A. De Maddalena, E. Rotelli, G. Barbarisi (eds.), Economica, istituzioni, cultura in Lombardia nell'età di Maria Teresa, vol. II, Cultura e società, Bologna, II Mulino, pp. 845-861

Levati S., 1997: La nobiltà del lavoro. Negozianti e banchieri a Milano tra Ancien Régime e Restaurazione, Milano, FrancoAngeli

Levati S., 2000: Notai e società nello Stato di Milano alla fine dell'antico regime 
(1751-1800): reclutamento, strategie familiari e ruolo sociale di un gruppo professionale, in M. Meriggi e A. Pastore (ed.), Le regole dei mestieri e delle professioni. Secoli XV-XIX, Milano, FrancoAngeli, pp. 120-151

Levati S., 2017: Fortune e clientela di un notaio tra Milano e Inzago: il caso di Ignazio Baroggi (1797-1841), in P. Grillo, S. Levati (eds.), Legittimazione e credito tra medioevo e ottocento. Notai e ceto notarile tra ruoli pubblici e vita privata, Milano, FrancoAngeli, pp. 231-244

Liva A., 1979: Notariato e documento notarile a Milano. Dall'alto medioevo alla fine del Settecento, Roma, Consiglio Nazionale del Notariato

Lombardi D., 2008: Storia del matrimonio. Dal Medioevo a oggi, Bologna, II Mulino

Mangini M.L., 2005: "Membra disiecta" del Collegio notarile di Como. Notai e forme di organizzazione della professione notarile in Valtellina e nel Bormiese (secc. XV ex.-XVI ex.), in "Bollettino della Società Storica Valtellinese", 58, pp. 149-194

Mazzanti Pepe F. - Ancarani G., 1983: Il notariato in Italia dall'età napoleonica all'Unità, Roma, Consiglio Nazionale del Notariato

Meriggi M., 1981a: Potere e istituzioni nel Lombardo-Veneto pre-quarantottesco, in P. Schiera (ed.), La dinamica statale austriaca nel XVIII e XIX secolo, Bologna, II Mulino, pp. 207-245

Meriggi M., 1981b: Liberalismo o libertà dei ceti? Costituzionalismo lombardo agli albori della Restaurazione, in "Studi Storici", 22, pp. 315-343

Meriggi M., 1983: Amministrazione e classi sociali nel Lombardo-Veneto (18141848), Bologna, II Mulino

Meriggi M., 1987: I/ Regno Lombardo-Veneto, in Storia d'Italia diretta da G. Galasso, vol. XVIII, p. II, Torino, Utet

Meriggi M., 2002: Gli Stati italiani prima dell'Unità. Una storia istituzionale, Bologna, II Mulino

Montorzi M., 1983: Il notaio di tribunale come pubblico funzionario: un primo quadro di problemi, e qualche spunto analitico, in "Rivista del Notariato. Rassegna di diritto e pratica notarile", 37, n. 6, pp. 1090-1128, nonché in II notariato nella civiltà toscana, Roma, Consiglio Nazionale del Notariato, 1985, pp. 7-59

Montorzi M., 1997: Giustizia in contado. Studi sull'esercizio della giurisdizione nel territorio pontederese e pisano in età moderna, Firenze, Edifir, pp. 132-141

Padoa Schioppa A., 1980: Sul ruolo dei giuristi nell'età del diritto comune: un problema aperto, in D. Segoloni (ed.), II diritto comune e la tradizione giuridica europea, Atti del Convegno di studi in onore di Giuseppe Ermini (Perugia, 30-31 ottobre 1976), Perugia, Libreria universitaria editrice, pp. 155-166, ora in Id., Italia ed Europa nella storia del diritto, Bologna, II Mulino, 2003, pp. 293-301 
Padoa Schioppa A., 2003: Sull'avvocatura, in Id., Italia ed Europa nella storia del diritto, Bologna, II Mulino, pp. 301-312

Pagano E., 2001: Avvocati ed esercizio della professione legale in Lombardia nel secondo Settecento. I causidici collegiati di Milano, in "Rivista di Storia del Diritto Italiano", 74, pp. 355-418

Perelli Cippo R., 1982: «Notarii» e «secundi notarii» a Milano nel Duecento, in "Nuova Rivista Storica", 66, pp. 594-598

Petronio U., 1972: II Senato di Milano. Istituzioni giuridiche ed esercizio del potere nel Ducato di Milano da Carlo V a Giuseppe II, Milano, Giuffrè

Pino F., 1979: Patriziato e decurionato a Milano nel secolo XVIII, in "Società e storia", 5, pp. 339-378

Pontiggia G. e Rumi G. (ed.), 1988. Il tramonto di un regno: il Lombardo-Veneto dalla Restaurazione al Risorgimento (1814-1859), Milano, Cariplo

Roberti M., 1947: Milano capitale napoleonica. La formazione di uno Stato moderno 1796-1814, vol. II, Milano, Fondazione Treccani degli Alfieri per la storia di Milano

Salvi S.T., 2009-2010: Riformismo teresiano e conservazione degli atti notarili. L'istituzione del Pubblico Archivio a Milano nel XVIII secolo, in "Rassegna degli Archivi di Stato", nuova serie, 5-6, pp. 41-64

Salvi S.T., 2012a: Tra privato e pubblico. Notai e professione notarile a Milano (secolo XVIII), Milano, Giuffrè

Salvi S.T., 2012b: Due generazioni di notai nella Milano di fine Ancien Régime: Giuseppe e Vincenzo D’Adda, in "Historia et ius", 2, paper 10, all'url http:// www.historiaetius.eu

Salvi S.T., 2013a: Carcano, Francesco Maria, in Dizionario biografico dei giuristi italiani (XII-XX secolo), diretto da I. Birocchi, E. Cortese, A. Mattone, M.N. Miletti, vol. I, Bologna, II Mulino, p. 444

Salvi S.T., 2013b: D’Adda, Vincenzo, in Dizionario biografico dei giuristi italiani (XII-XX secolo), diretto da I. Birocchi, E. Cortese, A. Mattone, M.N. Miletti, vol. I, Bologna, II Mulino, p. 624

Salvi S.T., 2017: I notai milanesi nel XVIII secolo: un ceto "poliedrico", in P. Grillo e S. Levati (ed.), Legittimazione e credito tra medioevo e ottocento. Notai e ceto notarile tra ruoli pubblici e vita privata, Milano, FrancoAngeli, pp. 213-230

Salvi S.T., 2022: Notai di età moderna mediatori? Qualche riflessione sul ruolo del notaio nella Lombardia austriaca (XVIII secolo), in A. Bassani, M.L. Mangini, F. Pagnoni (ed.), Mediazione notarile. Forme e linguaggi tra Medioevo ed Età Moderna, Quaderni degli Studi di Storia Medioevale e di Diplomatica, Milano, Mondadori, in corso di stampa

Santoro M., 1998: Notai. Storia sociale di una professione in Italia (1861-1940), 
Bologna, II Mulino

Santoro M., 2004: II notariato nell'Italia contemporanea, Milano, Giuffrè

Scazzoso M., 1982: Le Scuole Palatine a Milano nell'età delle riforme, in A. De Maddalena, E. Rotelli, G. Barbarisi (eds.), Economica, istituzioni, cultura in Lombardia nell'età di Maria Teresa, vol. III, Istituzioni e società, Bologna, II Mulino, pp. 887-895

Sinisi L., 2006: Judicis oculus. II notaio di tribunale nella dottrina e nella prassi di diritto comune, in V. Piergiovanni (ed.), Hinc publica fides. II notaio e l'amministrazione della giustizia, Atti del Convegno internazionale di studi storici, Genova 8-9 ottobre 2004, Milano, Giuffrè, pp. 215-240

Sinisi L., 2008: Aspetti dell'amministrazione della giustizia "in criminalibus" a Genova in età moderna, in Tra diritto e storia. Studi in onore di Luigi Berlinguer promossi dalle Università di Siena e di Sassari, vol. II, Soveria Mannelli, Rubbettino, pp. 1039-1056, nonché in A. Assini, P. Caroli (eds.), Spazi della memoria storica. La storia di Genova attraverso le vicende delle sedi e dei documenti dell'Archivio di Stato, Atti del convegno internazionale Genova, 7-10 giugno 2004, Genova, Ministero per i beni e le attività culturali, 2009, pp. 185200

Sinisi L. (ed.), 2011: Le leggi notarili. Dagli Stati preunitari al Regno d'Italia (18051879), Padova-Torino, Cedam-Utet

Sinisi L., 2012: Sviluppo ed evoluzione ottocentesca degli ordinamenti notarili italiani sino all'Unità, in "Rivista di Storia del Diritto Italiano", 85, pp. 41-108

Sinisi L., 2014: II notaio e la sua presenza nell'apparato giurisdizionale: profili storici, in La modernità degli studi storici: principi e valori del Notariato, Atti del convegno, Genova 16 maggio 2014, Quaderni della Fondazione del Notariato, 2 (2014), pp. 102-115

Sinisi L., 2020: Fra giurisdizione e documentazione. II notaio in tribunale dall'antico regime all'età dei codici, in "Iurisdictio", 1, pp. 221-247

Tavilla E., 2013: Da notaio di città a notaio di Stato: la normativa nel ducato estense (secc. XVII-XIX), in G. Tamba, E. Tavilla (eds.), Nella città e per la città. I notai a Modena dal IX al XX secolo, Milano, Giuffrè, 2013, pp. 143-180

Visconti A., 1922: La cattedra di diritto municipale e provinciale nelle Scuole Palatine e la soppressione delle Canobbiane, in "Archivio Storico Lombardo", 49, fasc. 1-2, pp. 3-14

Visconti A., 1927: Le Scuole Palatine di Milano, Milano, La Famiglia Meneghina Zaghi C., 1989: L'Italia di Napoleone, Torino, Utet

Zorzoli M.C., 2001: Alcune considerazioni sui collegi dei giuristi nella Lombardia d'antico regime, in "Annali di Storia moderna e contemporanea", 7, pp. 449-475

Zorzoli M.C., 2014: L'educazione del giurista per la pratica (nello Stato di Milano 
tra Cinque e Seicento), in P. Maffei, G.M. Varanini (eds.), Honos alit artes. Studi per il settantesimo compleanno di Mario Ascheri, vol. I, La formazione del diritto comune, Firenze, Firenze University Press, pp. 291-306

Fonti

ASMi, Collegio dei notai e dei causidici, cartella 236

ASMi, Dispacci reali, cartella 202

ASMi, Dispacci reali, cartella 203

ASMi, Dispacci reali, cartella 205

ASMi, Dispacci reali, cartella 209

ASMi, Dispacci reali, cartella 251

ASMi, Dispacci reali, cartella 254

ASMi, Giustizia punitiva parte antica, cartella 5

ASMi, Notarile, cartella 46551, notaio Maggi Gaetano quondam Benedetto

ASMi, Notarile, cartella 50558, notaio Mangiagalli Ambrogio quondam Luigi

ASMi, Studi parte antica, cartella 166

ASMi, Studi parte antica, cartella 168

ASMi, Studi parte antica, cartella 169

ASMi, Sudi parte moderna, cartella 319

ASMi, Studi parte moderna, cartella 320

ASMi, Uffici e tribunali regi parte antica, cartella 261

Giovanni Battista Bianchini, Compendium ordinum, stilatuum, et aliarum scripturarum decorem, et splendorem Ven. Collegii DD. Causidicorum et Notariorum Mediolani ostendentium, Mediolani, ex typographia Federici Francisci Maiettae, 1701

Francesco Maria Carcano, Il Notajo istruito colli schiarimenti del regolamento sul notariato e delle leggi analoghe e colle module degl'istromenti ..., Milano, dalla Società Tipografica de' Classici Italiani, 1810

Vincenzo D'Adda, Arte notarile in tre parti divisa, Milano, Presso Giuseppe Taglioretti al Cordusio, 1796

Gridario dal 1727 al 1794, Milano, G.R. Malatesta [1727-1794], vol. VIII

Daniele Lissoni, Progetto di legge per l'esercizio del Notariato con annotazioni, cenni storici e raffronti, Milano, Tip. Boniardi-Pogliani di Ermen. Besozzi, 1868

Ordines pro Notariis et Collegio Causidicorum, MDCLXXXVI, Die xxii Augusti, in Ordines Excellentissimi Senatus Mediolani ab anno MCDXC usque ad annum MDCXXXIX collecti, et scholiis ornati ab olim J.C. Angelo Stephano Garoni ..., Mediolani, in Curia regia sumptibus Joseph Richini Malatestae regii ducalis 
typographi, 1743

Regolamento sul Notariato, 17 giugno 1806, Milano, dalla Stamperia Reale, 1806, altresì in Bollettino delle leggi del Regno d'Italia, Milano, 1805-1814, 1806, parte II, pp. 664-717

Pietro Verri, Orazione panegirica sulla giurisprudenza milanese, in F. Venturi (ed.), Cesare Beccaria. Dei delitti e delle pene, con una raccolta di lettere e documenti relativi alla nascita dell'opera e alla sua fortuna nell'Europa del Settecento, Torino, Einaudi, 1970, pp. 144-145 